\title{
Representaciones de la otredad: experiencia femenina e identidad en ¡Negras somos!
}

\author{
Representations of Otherness: Feminine Experience \\ and Identity in ${ }_{i}$ Negras somos! \\ Representações da outredade: experiência feminina \\ e identidade em ;Negras somos!
}

\section{M'bare N'gom}

MORGAN STATE UNIVERSITY, ESTADOS UNIDOS

Profesor del Department of World Languages and Graduate Program in International Studies. Director de los programas de Estudios Africanos y Estudios Latinoamericanos y Caribeños. Doctor en Letras, Universite de Paris-Sorbonne (Paris IV). Autor de Diálogos con Guinea Ecuatorial: Panorama de la literatura guineoecuatoriana de expresión española a través de sus protagonistas (Ediciones LABRYS 54, 1996), Palabra Abierta: Entrevistas con los escritores africanos hispanos (Verbum, 2013) y editor de La reconstrucción de la memoria y de la identidad nacional en la literatura hispanoafricana (Universidad de Alcalá de Henares, 2004), "Escribir"la identidad: creación cultural y negritud en el Perú (Fondo Editorial de la Universidad Ricardo Palma, 2008). Correo electrónico: mbare.ngom@morgan.edu 


\section{Resumen}

Este trabajo se propone examinar, de forma específica, la literatura colombiana producida por mujeres de ascendencia africana desde la periferia del llamado "canon literario", y que nosotros definimos como institucionalidad cultural oficial. Para ello, nos apoyaremos en el texto $; N$ Negras Somos! Antología de 21 mujeres poetas afrocolombianas (2008), una de las primeras colecciones de su género publicada en Colombia y quizás en Latinoamérica, dedicada exclusivamente a la producción cultural de las mujeres escritoras de ascendencia africana. Por un lado, se examinará el discurso lírico como plataforma de recuperación y de construcción de un espacio de expresión personal y colectiva y, por el otro, como medio de afirmación de identidad, pero de una identidad multifacética: femenina, negra y colombiana; en otras palabras, la experiencia de "ser-mujer y negra" dentro de un espacio nacional y de soberanía internacional llamado República de Colombia.

Palabras clave: Afrocolombianas; historiografía cultural oficial; voces femeninas; transafricana; talking back.

\section{Abstract}

The purpose of this work is to examine specifically Colombian literature produced by Africandescent women, from the periphery of the so-called "literary cannon", which we defined as Official Cultural Institutionality. To this end, we will base our work on the text 'iNegras Somos!' Anthology of 21 afro-Colombian poetesses (2008) -one of the first collections in its genre published in Colombia, and maybe even in Latin America- which is dedicated exclusively to the cultural production of female writers of African descent. On the one hand, we will examine the lyrical discourse as a platform for the recovery and construction of a personal and collective space for expression; on the other hand, we will examine it as a means for the affirmation of identity, a multifaceted identity: feminine, black, and Colombian. In other words, the experience of 'being black and female' in a national space, with international sovereignty, called Republic of Colombia.

Palabras clave: Afrocolombianas; historiografía cultural oficial; voces femeninas; transafricana; talking back.

\section{Resumo}

Este trabalho propõe-se examinar de forma específica a literatura colombiana produzida por mulheres de ascendência africana desde a periferia do chamado de "cânone literário" e que nós definimos como institucionalidade cultural oficial. Para isso, apoiarnos-emos no texto ;Negras Somos! Antología de 21 mujeres poetas afrocolombianas (2008), uma das primeiras coletâneas do seu género publicada na Colômbia e talvez em Latino América, dedicada exclusivamente à produção cultural das mulheres escritoras de ascendência africana. De um lado vai se examinar o discurso lírico como plataforma de recuperação e de construção de um espaço de expressão pessoal e coletiva e do outro lado, como meio de afirmação de identidade, mais de uma identidade multifacetada: feminina, negra e colombiana; em outras palavras, a experiência de "ser-mulher e negra" dentro de um espaço nacional e de soberania internacional chamado de República da Colômbia.

\section{Palavras-chave:}

Afrocolombianas; historiografia cultural oficial; vozes femininas; transafricana; talking back

\section{Cómo citar este artículo:}

$\mathrm{N}^{\prime}$ gom, M'bare. "Representaciones de la otredad: experiencia femenina e identidad en ¡Negras somos!”. Cuadernos de Literatura 19.38 (2015):

119-136. http://dx.doi.org/10.11144/Javeriana.cl19-38. roef 
LA LITERATURA COLOMBIANA escrita por transafricanos, africanos y sus descendientes, ha recibido una atención teórica y crítica limitada al estar centrada en unos pocos escritores considerados "clásicos" o "canónicos" como Candelario Obeso, Manuel Zapata Olivella, Jorge Artel, Arnoldo Palacios y Juan Zapata Olivella, entre otros. En cambio, los proyectos culturales producidos por mujeres colombianas de ascendencia africana, no solo brillan por su ausencia, sino que parecen "inexistentes" tanto desde el punto de vista editorial como de la crítica. En este sentido, si bien hasta 2011 la historiografía cultural oficial había relegado la literatura afrocolombiana -entiéndase la producida por hombres- a espacios periféricos a lo que Pierre Bourdieu llama "le champ littéraire" (el campo literario). Dentro de ese marco, la producción cultural de las mujeres transafricanas estaba marcada por el sello de la invisibilidad cultural, editorial, crítica y académica ${ }^{1}$.

Este trabajo se propone examinar, de forma específica, la literatura colombiana producida por mujeres de ascendencia africana desde la periferia del llamado "canon literario" y que nosotros definimos como institucionalidad cultural oficial. Para ello, nos apoyaremos en el texto ;Negras Somos! Antología de 21 mujeres poetas afrocolombianas (2008), una de las primeras colecciones de su género publicada en Colombia y quizás en Latinoamérica, dedicada exclusivamente a la producción cultural de las mujeres escritoras de ascendencia africana. Por un lado, se examinará el discurso lírico como plataforma de recuperación y de construcción de un espacio de expresión personal y colectiva y, por el otro, como medio de afirmación de identidad, pero de una identidad multifacética: femenina, negra y colombiana; en otras palabras, la experiencia de "ser-mujer y negra" dentro de un espacio nacional y de soberanía internacional llamado República de Colombia. Partiendo de esas premisas, cabe preguntarse, ¿qué significa o representa la experiencia de "ser-mujer y negra" en una realidad que niega la realidad de ese "ser-mujer y negra" definida entre otras circunstancias, por la compleja y dinámica interacción e intersección entre género, clase, raza, ciudadanía, y mediada por una opresión doble. Partiendo de esas premisas, cabe

1 Usamos el término transafricano en el mismo sentido que afrodescendiente o de ascendencia africana.

2 Aquí partimos del concepto de "ser mujer" propuesto por Ana María Agudelo Ochoa, quien lo define como una "categoría construida en el marco de un pensamiento controlado por las instituciones que manejan el campo de poder, bajo los preceptos de la moral cristiana, la política conservadora y una estructura social donde el hombre ocupa un lugar superior, determina el discurso crítico e historiográfico, asimismo legitima y valora la producción artística de las mujeres en la medida en que se ajuste a tales lineamientos" (92). 
preguntarse, ¿qué lugar ocupa la mujer, en general, como creadora de múltiples discursos epistemológicos dentro de un territorio socio-cultural e institucional dominado por la visión hegemónica y patriarcal criolla en América Latina? De entrada, es preciso recordar el protagonismo silenciado de la mujer en la realidad histórica, social, económica y cultural de las Américas. Su experiencia historiográfica ha sido marcada por una presencia-ausencia o presencia negada, durante las fases de exploración y de conquista del territorio continental e insular que los europeos llamaron América. La mujer fue borrada de los textos que recogían aquella experiencia histórica. Durante la época colonial la mujer fue rescatada y representada como sujeto literario activo y autónomo gracias a una de las escasas voces femeninas de entonces: Sor Juana Inés de la Cruz (16481695). A esa voz "solitaria", es preciso sumar la voz mitigada de Sor Ursula de Jesús (1604-1666), una esclava "donada", sirviente de una dama principal. Ursula sirvió unos 28 años en un convento de Lima en el Perú. Ursula de Jesús es, sin lugar a dudas, al menos de momento, la primera mujer de ascendencia africana que invade el espacio letrado en Hispanoamérica. Salvo contadas ocasiones, la presencia continua y sostenida de la mujer dentro del marco historiográfico cultural latinoamericano, así como su experiencia personal, han sido, hasta mediados del siglo XX, aquella que fue recogida en las obras literarias dentro de un marco crítico e historiográfico regentado por el discurso hegemónico colonial y más tarde, el republicano criollo. La perspectiva que emanaba de esas prácticas culturales estaba fuertemente contaminada por una percepción y representación de la realidad profundamente mediada, en primera instancia, por una visión patriarcal europea. Luego esa perspectiva fue heredada por el grupo hegemónico criollo que usó los mismos parámetros para definir a la mujer que seguía siendo un ser periférico, fragmentado, "vulnerable" y posicionado en espacios enclaustrados. Dentro de ese marco histórico, político y cultural, la mujer carece de voz y de autonomía, ya que solo puede expresarse de forma legítima y afirmar su presencia gracias a la mediación del discurso hegemónico del patriarcado criollo. Marcia Welles (1996) observa a tal efecto que: "The portrayal of women by male authors tends to facile stereotypes drawn more from the dimension of myth than from that of actuality" (280).

La mujer representada y que opera a partir de esos parámetros parece incapaz de articular un discurso autónomo aceptable y aceptado, lo cual le impide construir una identidad femenina auténtica sin la mediación del discurso patriarcal. Extrapolando al resto de la literatura de América latina durante el mismo período, Margarita Vargas (1997) observa que, "Similar negative representations of women during the Romantic period can be found in the literatures of other 
Spanish-American countries" (3). La visión que se desprende de ello es la de una experiencia femenina falsificada, contaminada y por ende, inauténtica al apoyarse en y al partir de una identidad construida a base de estereotipos racializados. Ese discurso de la masculinidad nos da una perspectiva alienante y reduccionista que algunas mujeres incluso llegaron a asumir como propio, como en el caso de la escritora colombiana Agripina Montes del Valle en 1868: "La literatura, ese sueño de la mujer espiritual y sensible, no puede realizarse cuando ella ha contraído deberes tan sagrados como los del hogar" (314).

De-responsabilizada y posicionada al margen de la historia nacional y de la construcción del discurso de la identidad nacional en muchos países de América latina, la mujer se convirtió, al menos en los espacios públicos de transacción, en una protagonista marcada por el sello de la invisibilidad cultural, política y económica, así como del silencio. En cuanto a las mujeres procedentes de las minorías sociales, étnicas y raciales como las transafricanas, negras y mulatas, por un lado y las de los pueblos originarios por el otro, se encontraban doblemente marginadas en comparación con la mujer criolla. La mujer negra, limitándonos al caso que nos ocupa aquí, era integrante de un grupo racial de entrada descentrado por el discurso hegemónico colonial blanco y más tarde por el republicano y criollo y como no, por la mujer criolla. Convertida en objeto presente-ausente según las necesidades sociales, la mujer negra "fue ausentada", al menos oficial y públicamente, del proceso de construcción del Estado-nación en muchos países latinoamericanos. Definida por la presencia/negación, la experiencia femenina transafricana tenía menos posibilidades de ser recogida y luego expresada de forma fidedigna en los espacios públicos e institucionales de transacción. Claudette Rose Green Williams (1993) nota que

One of the most durable and damaging stereotypes propagated in the poetry of Spanish speaking Caribbean is that of the woman of African descent as an ontologically sensual creature. Many poets are irresistibly driven to write about the sexual attributes and behaviour of the Afro-Caribbean woman, and, in fact sexuality is the main context in which they defined her identity. (16)

El advenimiento del movimiento feminista occidental organizado a principios del siglo XX tampoco cambió mucho dicha visión. Si bien el objetivo del feminismo de entonces era extraer a la mujer de la estructura de la marginación donde había sido confinada, este contemplaba más bien la situación de la mujer criolla. En efecto el movimiento feminista procedente de Occidente no supo ni pudo alcanzar el nivel de sensibilidad y de solidaridad que le permitiera incluir en su agenda ideológica la realidad socio-cultural, política y económica de las 
mujeres americanas de ascendencia africana. Las circunstancias históricas y socio-culturales de la mujer negra eran diferentes y ajenas a las del movimiento feminista que era fuertemente eurocéntrico ${ }^{3}$. La mujer transafricana se quedó estancada en la precariedad del silencio y de la marginación social, política, económica y sobre todo en la estructura de la pobreza. Willy O. Muñoz (1999) recalca en este sentido que

El esencialismo que el feminismo adoptó en un primer momento ignoró la peculiar problemática de las mujeres de color para codificar exclusivamente la condición de la mujer blanca de la clase media. Si bien hoy la teoría incluye en sus postulados a la mujer negra, en la práctica todavía quedan residuos discriminatorios en las mujeres blancas. (217)

Mientras la mujer integrante del grupo hegemónico criollo se iba incorporando a ciertos espacios públicos de transacción y de expresión, la mujer negra ,en cambio, se hundía cada vez más en una estructura de silencio, de otredad doble, de violencia y por ende, de invisibilidad. Rosemary Geisdorfer Feal (1991) nota al respecto: [...] The oppression of women-black women in particular, but all Latin American women-accurately reflects the racist patriarchal structure of the societies out of which these works come. (21)

En 1864, la autora colombiana Agripina Samper reclamaba precisamente un espacio de expresión dentro del marco de la institucionalidad cultural oficial en un emblemático ensayo titulado, "¿Por qué no he de escribir yo también?", y publicado en la revista El Mosaico (1864) (ctd en Agudelo Ochoa 9o). Su protesta, aunque colectiva, no contemplaba, ni mucho menos, a esas mujeres invisibles como la negra y la de los pueblos originarios. Su voz expresaba, sin lugar a dudas, el sentir de un sector de la mujer criolla, pero cuando la Ciudad Letrada, por usar el término de Ángel Rama, empieza a recoger la "palabra de la mujer" (Navia Velasco, 163), esa iniciativa no contempla ni incluye a la mujer transafricana colombiana.

3 Khadijah Bawazeer (2013), confirma el dilema del movimiento ante las realidades ajenas de las llamadas "sociedades periféricas" o "subdesarrolladas". Y dice: "At home, white western women theories are open to interpretations, modification, and evolvement. However, when transported, they are treated as hard judgemental blocks. Abroad, physically and metaphorically, the theories and practices of women's movements become closed and finalized models that are not open to deconstruction and reshaping. Sometimes, they are imposed on societies by its elites regardless of their suitability" (51). 
La historiografía literaria colombiana revela una ausencia notable de las autoras colombianas de ascendencia africana de los espacios culturales de la institucionalidad cultural oficial. Si bien hubo iniciativas de divulgación de los procesos creativos de la mujer en Colombia, la autora afrocolombiana ha sido, por decirlo de alguna forma, la convidada de piedra. En 1975, Eddy Torres publica Poesía de autoras colombianas, una antología que recoge las selecciones de siete poetas del periodo colonial y republicano y treinta autoras contemporáneas entre 1901 y 1975. No recoge ni menciona la presencia afrocolombiana (ctd en Cuesta, 13). En 1995, Teresa Rozo-Moorhouse saca la antología Diosas en bronce, la cual incluye a tres poetas afrocolombianas de un total de 97: Nadhyma Triana (1932), María Teresa Ramírez (1944) y Ana Milena Lucumi (1964)4 .

La antología que nos ocupa en este trabajo, con su título contundente y provocador, ¡Negras somos!, es una colección de productos líricos y narrativos por 21 poetas colombianas de ascendencia africana. Publicada en el 2008 por el programa editorial de la Vicerrectoría de Investigaciones de la Universidad del Valle, la colección fue compilada por los poetas Guiomar Cuesta Escobar y Alfredo Ocampo Zamorano y tiene un marcado carácter regional al limitarse o enfocarse en el Pacífico Colombiano. Si bien el libro marca un hito en la historiografía literaria de Colombia y de Hispanoamérica, en general, no contempla la producción cultural de todas las autoras nacionales, por lo tanto sería problemático adjudicarle un sello nacional representativo de toda la afrocolombianidad literaria en general y de la femenina en particular.

La obra se apoya en una periodización flexible estructurada entorno al año de nacimiento de las autoras. El libro consta de tres grandes partes o capítulos:

- Nacidas antes de 1950;

- Nacidas en la década de los años cincuenta;

- Nacidas en la década de los sesenta y setenta.

Si bien algunas de las autoras recogidas aún siguen activas, existe un lapsus de unos treinta años que hace falta colmar. Y es precisamente lo que han hecho los mismos compiladores de la colección aprovechando la laudable iniciativa del Ministerio de Cultura de la República de Colombia. En 2011, durante el gobierno del Presidente Álvaro Uribe, coincidiendo con la celebración del Año Internacional de los Afrodescendientes decretado por la Organización de las Naciones Unidas, el Ministerio de Cultura de Colombia lanzó la iniciativa de la publicación de lo que se podría considerar como una obra magna: la Biblioteca de la Literatura Afrocolombiana que

4 Sobre esa trayectoria véase la introducción en ;Negras Somos! Antología de 21 mujeres poetas afrocolombianas (4-45). 
[...] contiene 18 títulos y un ensayo escritos por autores Afrocolombianos/as destacados en los últimos 200 años; entre ellos están las importantes obras de autores como: Manuel Zapata Olivella, Oscar Collazos, Helcías Martán Góngora, Arnoldo Palacios, Rogerio Velásquez, Candelario Obeso, entre otros. La relectura y re-significación de la literatura nacional, pasa por la visibilización y el reconocimiento de los aportes que los autores Afrocolombianos/as han realizado, a través de los importantes procesos de resistencia cultural que bajo el proyecto de libertad ejercido en épocas coloniales y contemporáneas se configuran en la consistencia y la valiosa capacidad creativa que en todas las áreas del arte poseen los descendientes de las diversas culturas africanas llegadas a Colombia". (Cuesta y Ocampo Introducción).

El volumen XVI de la colección, titulado, Antología de Mujeres Poetas Afrocolombianas, "amplía y completa la anterior, ya que se ocupa de las poetas afrocolombianas de todo el país" (Cuesta y Ocampo. Antología 11), según afirman los compiladores. En efecto, estos procedieron a la ampliación y a la re-estructuración del texto original por medio de una periodización más específica y al incluir tanto a autoras de la época republicana temprana como de otras contemporáneas y procedentes de todo el territorio nacional:

- Las pioneras: nacidas antes de 1940;

- Nacidas en la década de 1940;

- Nacidas en la década de 1950;

- Nacidas en la década de 1960;

- Nacidas en la década de 1970;

- Nacidas en la década de 1980;

Al ser descentradas y desterritorializadas de la ciudad letrada y excluida de la historiografía literaria colombiana, las creadoras culturales afrocolombianas encontraron un espacio de expresión en el seno del Encuentro de Poetas Colombianas, cuya primera edición se celebró en la ciudad de Rodanillo, Valle del Cauca, en 1984, "el único espacio en Colombia donde se respeta la voz de las mujeres poetas afrocolombianas, en todo lo que ellas aportan de novedoso a la poesía de la mujer, y a la poesía colombiana en particular" (Cuesta 14). Según observan los compiladores, once de las veintiuna integrantes de la antología ;Negras somos! han participado en los encuentros de Cali durante los últimos 24 años. Ese foro les sirvió de trampolín para asomarse al espacio cultural institucional de la ciudad letrada. Ahora bien, eso no significa ni mucho menos que fueran aceptadas tanto dentro como fuera de aquel espacio.

El proyecto lírico/narrativo de las mujeres, cuyos trabajos son recogidos en la antología intenta deconstruir la narrativa institucional de la ciudad letrada 
mediada por el racismo, el sexismo, la discriminación y la violencia de género. Por otro lado, intenta abrogar el silencio sistemático y sostenido hacia la mujer autora de ascendencia africana y proponer vías discursivas y críticas alternativas en su aproximación a la representación de su(s) experiencia(s). Muchos de los poemas se erigen contra un país oficial que excluye y oprime a la mujer transafricana que es expuesta y sufre distintos niveles y grados de violencia.

Por lo tanto, la práctica de "ser-mujer y negra", tal como se expresa en la obra, busca la superación de la cultura del silencio impuesto y de la presencianegada, no solo por la institucionalidad cultural oficial, sino también dentro de la misma comunidad transafricana, también dominada por la visión patriarcal. $\mathrm{Y}$ ello se refleja en ese empeño de las autoras de la antología en abrogar lo que Carol Boyce-Davies llama voicelesness y, de paso, liberar y recuperar "esa voz perdida" a la que hacía referencia el poeta dominicano Blas Jiménez Abreu. Las poetas de ¡Negras somos! intentan desatar y soltar esa voz ahogada y cómo no, romper las estructuras que las confinaban como mujeres y negras a espacios determinados de antemano por los diversos discursos patriarcales: por un lado, el espacio regentado por el patriarcado hegemónico criollo y por el otro lado, el que prevalece dentro de su propio espacio étnico e íntimo del que procede. Por lo tanto, el "ser-mujer y negra", tal como lo entienden y expresan las autoras de la antología a través de sus textos, es una postura de empoderamiento, de validación y de legitimación que les permite replicar "de tú a tú" y de forma contundente y efectiva al modelo discursivo del patriarcado. La crítica estadounidense bell hooks define esa actitud como talking back, porque, además de asentarse en la autonomía de la voz propia, le permite a la mujer negra denunciar la discriminación y los diversos niveles de abusos de los que ha sido objeto. Es en última instancia, una postura de auto-rescate del enclaustramiento y de rechazo de lo que el crítico Victorien Lavou describe como la memoria imposée. Y bell hooks observa al respecto:

Moving from silence into speech is for the oppressed, the colonized, the exploited, and those who stand and struggle side by side, a gesture of defiance that heals, that makes new life and growth possible. It is that act of speech of "talking back", that is no mere gesture or empty words, that is the expression of our movement from object to subject-liberated voice. (ctd en Muñoz 245)

La postura ideológica de poder que representa el hecho de talking back conlleva la articulación de una actitud y una disposición discursiva que Carol Boyce-Davies llama naming or re-marking. Ello implica y demuestra la capacidad de la mujer transafricana de conformar un lenguaje identitario propio sin ambigüedades que le permita reconstruirse textualmente como mujer, madre, 
ciudadana y amante si cabe, dentro del proyecto nacional. Se trata pues, de una de-construcción del modelo histórico patriarcal prevalente sobre la mujer negra y transafricana. Ello implica la de-construcción del cuerpo construido e impuesto, como suyo, a través de una multitud de discursos ajenos. Ese proceso implica una transgresión de las normas societales reservadas a las mujeres criollas y la adjudicación de una nueva estructura de poder, empoderamiento, se diría por los tiempos que corren, a partir de la cual se movería la mujer transafricana. Al mismo tiempo, esas escritoras colombianas invaden el espacio de poder patriarcal cuya masculinidad, fuente y justificación de su dominio, cuestionan. El proyecto de retextualización de la mujer y de la mujer transafricana en particular, empieza con su liberación personal y la recuperación del cuerpo propio, su redescubrimiento y el goce del mismo desde la autonomía. Así lo recoge Elisa Rosada de Pupo en el poema "Posesión":

Soy la dueña

de la trampa

que encierra mis sentidos

del vértigo que succiona

y atrapa mi existencia

Silencio desconfianza;

También la encontramos en "Cuerpo erótico" de Ana Teresa Mina Diáz,

La tibieza de tu piel color canela

Sacude la sutileza silvestre de mi vientre

Se estremece el cortejo de mis labios

Libando el polvillo de las flores;

O en "Esta noche" de Sobeida Delgado Mina, de la generación de los 80

Quiero navegar en el mar

De tu cuerpo desnudo,

Para posarme

En la punta

De tu montana,

Beber el néctar

De tu esencia varonil

Y sumirme

En lo mas profundo

De tu orgasmo 
La postura (ideológica) transgresiva de ese discurso se convierte en última instancia, en una actitud que de-legitima la memoria impuesta y por ende, el discurso histórico de representación de la mujer afrocolombiana, en el caso que nos ocupa. La reposesión del cuerpo propio es una forma de reposicionamiento y de codificación discursiva que recoge lo que Edna Acosta-Belén llama "women's participation in social movements and new ways of representing women's voices"(9). Asimismo ¡Negras somos! forma parte pues, de un amplio proyecto de de-construcción de los valores y de las prácticas epistemológicas del discurso patriarcal enfocado a perpetuar la otredad periférica de la negritud femenina en general. Asimismo la antología se erige e integra un proceso de re-ubicación histórica, social, política y cultural de la mujer transafricana que Asunción Lavrin describe en estos términos:

The ongoing project of historical reconstruction of the roles of all women and men of color and their participation in the building of their respective societies is now providing a voice to those sectors of society previously deprived of the power for formulating and transmitting knowledge. (6)

Las voces que recorren los textos de ;Negras somos! proponen "alternative ways (...) of developing appropriate constructs or making decisions about the relevance of existing ones" (Rice Murray 86), y conforman una memoria colectiva que se abre un espacio en el coto cerrado de la ciudad letrada patriarcal para decir la experiencia de "ser-mujer y negra". Al arrogarse el poder y el derecho de decir la experiencia histórica propia, dichas autoras proceden a la reconceptualización del "ser-mujer y negra" ya no como un "objeto sin historia" (Said 65-74), es decir un sujeto física y conceptualmente subyugado, sino como "sujeta" hacedora de historia. Proponen una lectura íntima y activa de las dinámicas culturales, intelectuales y políticas de la mujer negra cuya memoria liberan para que pueda decir su Historia y crear un espacio de solidaridad. En "Negra soy" Mary Grueso Romero dice,

¿Por qué me dicen morena?

Si moreno no es color

Yo tengo una raza negra

Y negra me hizo Dios;

y en "Orishas", de la misma autora,

Estoy tras los caminos

De mi identidad

Buscando las huellas 
De mis ancestros

El carimba me habla de África

Y después perdí el rastro

Cuando las olas despeinadas

Fueron tocadas en los mares

Por la mano azul del viento. (80)

"Dinga y Mandinga", de María Teresa Ramírez

Tengo dinga en mi sortija, el mandinga en mis aretej,

el Yoruba en mi cintura y el Congo en mi canalete, cuando voy rema que rema, por el rio de la vida

"Recobrando el pasado", de Dionicia Moreno Aguirre

Déjame ser negra de corazón.

Déjame sentir henchida el alma de alegría de angustia

De orgullo y de pasión

Ver a mis viejos, viejos...De ancestralidad,

Ver a mis viejos masticando el dolor de otros tiempos,

Déjame remontar a mis raíces

La recuperación de la "voz perdida" por medio de la re-articulación de esa identidad doblemente ahogada por medio de la re-historización de un pasado banalizado por la historia nacional se da por medio de la recreación de lo que el crítico congolés Pius Ngandu Nkasama llama douleur commune (dolor común), define la experiencia transafricana de las Américas. El dolor es físico en "Zumbo Zurungo" de Mary Grueso Romero,

Cuzumbo zumbo zunungo

Palabras

amargas son

Pronuncia el negro coplero

Ardido de fiebre y sudor

Delirando por la malaria

Que en los raiceros pescó;

No pescó más que miseria

Enfermedad y dolor; 
Es psicológico en "Siempre presentes", de Lorena Torres Herrera,

Quisieron borrar nuestras huellas

quisieron silenciar nuestras voces,

pero el cuerpo, cansado, desnudo

y maltratado por el látigo....!volvió a levantarse!

Quisieron borrar nuestra historia,

quisieron borrar nuestra imagen,

pero el alma, dolida, insistente

Y curtida

¡volvió a reescribirse! (185)

y en "Carimba", de Lucrecia Panchano, se trata de la agresión y degradación del cuerpo negro,

Carimba. Marca de abominable esclavitud

Que todo nos robó excepto la conciencia

Que en nosotros releva su física presencia

Y enfatiza en el negro, su máxima virtud.

Carimba...marca indignante del vasallaje

Que quiso destruir todas nuestras raíces.

$\mathrm{Y}$ aunque hoy presentamos diferentes matices.

Somos supervivientes de infame coloniaje. (51)

El recurso al panteón de las deidades africanas, sobretodo yoruba, es otra expresión de esa conciencia de "ser-mujer y negra" a partir de la cual puede proclamar su identidad como ciudadana, mujer, madre, compañera, amante y amiga al tiempo que proponen nuevas actitudes ante la vida y otra forma de decir la experiencia femenina. Asimismo, puede redefinir y replantear esa propuesta de diálogo a partir de nuevas normas de interacción con la sociedad. Mary Grueso Romero lo resalta en "Orishas"

En una noche estrellada

De misterio y, liturgia y festín

Apareció Yemayá

La diosa de los mares.

Me ungió con agua salda

Y emergí como un volcaán

Frente a Changó, Agum, Abatatá,

Oxulá, Elegua, Alofi,

Omolú, Oba, Yanza (81). 
En de esos poemas hay una propuesta de refundación de la categoría de "ser-mujer y negra" cuyo propósito es el replanteamiento de las normas que definen el "campo literario" en Colombia y en Latinoamérica en general. La reconstrucción de la realidad histórica y socio-cultural de las "voces perdidas" a quienes intentan reincorporar a la ciudad letrada y por ende a la historia oficial, se inscribe en ese marco. En este sentido, tanto la Biblioteca de la Literatura Afrocolombiana como la creación de la Cátedra de Estudios Afrocolombianos representan importantes iniciativas dentro de ese proceso incluyente ${ }^{5}$ ¡ $\mathcal{N e g r a s}$ somos! propone una re-textualización de un colectivo, la mujer transafricana, doblemente marginada e invisibilizada por la historiografía literaria en Colombia. A tal efecto, como observa Bernice Johnson-Reagan, "there is the opportunity to see a process of continuance and transformation at work among women cultural workers. Their struggle to contend with a new space entails defining their people and their children in new ways" (178).

¡Negras somos! como discurso de una memoria colectiva se propone re-definir para la sociedad colombiana, en el caso que nos ocupa, los rasgos esenciales que conforman la identidad femenina negra y más específicamente, la postura de "sermujer y negra". La subjetividad de la mujer transafricana desterrada de los espacios públicos de interacción y transacción y categorizada como objeto sexual, convirtieron su voz y perspectiva en modos discursivos irrelevantes condenándola al silencio. Privada de su capacidad pública de poner en tela de juicio los modos epistemológicos y sociales establecidos e impuestos por el sistema patriarcal, la mujer transafricana fue construida, definida y codificada como una ciudadana marginal cuya presencia es determinada por el valor de uso. Por lo tanto la perspectiva que emerge de ;Negras somos! está marcada por el compromiso y el afán por recuperar la parole perdue, en palabras de Edouard Glissant, para afirmar la identidad auténtica de la mujer transafricana, la cual, según la activista abolicionista norteamericana Sojourner Truth, implica ser "[...] a person who carves out a personal identity that culminates a culture of self-empowerment" (ctd en Jones 161). Esa actitud se manifiesta de varias maneras. En "Forma de vida" Elisa Posada de Pupo expresa autonomía como ser, mujer y ciudadana,

El tipo de vida con que sueño

es el que estoy viviendo

Jamás he permitido obstáculos y derrotas

Poseo la herramienta

5 La Cátedra de Estudios Afrocolombianos fue establecida por medio de la Ley 70 de 1993, concretada en el decreto 1122 de 1998. 
Para crear lo que quiero.

Esto es magia,

Bienvenida magia

a la vista de mis futuras generaciones (59);

mientras que en "Mi negritud" Jenny de la Torre Córdoba, resalta el orgullo racial

Mi negritud no se doblega,

Impetuosa como un huracán,

Insiste y penetra, no deja espacio

para la pena (127)

La afirmación de esa identidad supone articular un proceso que implica pasar de la categoría epistemológica de ser une femme écrite (una mujer escrita) a la de une femme écrivant (una mujer escribiendo), en palabras de la crítica Irêne d'Assiba Almeida (1991). Con ello, reclaman también un espacio de expresión y de solidaridad, así como el reconocimiento de sus derechos fundamentales básicos, la igualdad y una representación igualitaria y efectiva dentro del marco del Estado-nación.

En este sentido, ¡Negras somos! forma parte de un corpus literario más amplio y por descubrir. Codifica y de paso, critica y denuncia la opresión, marginación y trivialización de la que es víctima la mujer en general y la mujer negra en particular, la cual se enfrenta a diferentes tipos de discriminación, de violencia, así como a la tensión en casi todos los espacios donde evoluciona. Asimismo, además de intentar abrogar las relaciones asimétricas establecidas por el patriarcado colonial y luego heredadas y replicadas por el patriarcado criollo y más tarde republicano, ¡Negras somos! cuestiona la visión y postura céntricas, monolíticas y monoglósicas del patriarcado hegemónico republicano y no-hegemónico periférico, en algunos casos, en Colombia y América latina. El colectivo de voces que conforman ;Negras somos! propone una reformulación de las relaciones y de los discursos de poder al tiempo que ofrece perspectivas nuevas y alternativas sobre la mujer negra y su participación en la ampliación y consolidación de una colombianidad democrática y más abierta.

\section{Obras citadas}

Acosta-Belén, Edna. "Opening New Paths: Research on Women in Latin

America and The Caribbean". Woodrow Wilson International Center for

Scholars Working Papers. California: University of California, 1994. 2-36. 
Agudelo Ochoa, Ana María. "La literatura escrita por mujeres en una propuesta de aproximación histórica a la literatura colombiana". Tradiciones y configuraciones discursivas: historia crítica de la literatura colombiana. Elementos para la discusión. Cuadernos de trabajo II. Alfredo Laverde Ospina y Olga Vallejo Murcia Coords. Medellín: La Carreta Editores, 2010. 87-103.

Assiba d'Almeida, Irène. "L'écriture féminine en Afrique noire francophone. Le temps du miroir". Les Études Littéraires 24 (Automne 1991): 41-50. Assiba d'Almeida, Irene. Francophone African Female Writers: Destroying the Emptiness of Silence. Gainesville: University Press of Florida, 1994.

Bawazeer, Khadijah. "Feminism and the dilema of solidarity". Global Advanced Research. Fournal of Peace, Gender and Development Studies 2.3 (2013): 50-53.

Beverley, John. "Introducción". Revista de Crítica Literaria Latinoamericana 18.36 (1992): 7-18.

Bourdieu, Pierre. "Le champs littéraire". Actes de la Recherche en Sciences Sociales 89.89 (1991): 3-46.

Cuesta Escobar, Guiomar y Alfredo Ocampo Zamorano. "Prólogo". „Negras somos! Antología de 21 mujeres poetas afrocolombianas de la región Pacífica. Santiago de Cali: Programa Editorial de la Universidad del Valle, 2008: 13-45.

Cuesta, Guiomar y Ocampo Alfredo. Antología de mujeres poetas afrocolombianas. Bogotá: Ministerio de Cultura, 2010.

Delgado Mina, Sobeida. "Esta noche". ¡Negras somos! Antología de 21 poetas afrocolombianas de la región Pacífica. Alfredo Ocampo y Guiomar Cuesta. Comps. Bogotá: Ministerio de Cultura, 2008. 536-542 de la Torre Córdoba, Jenny. "Mi negritud”. „ iNegras somos! Antología de 21 poetas afrocolombianas de la región Pacífica. Alfredo Ocampo y Guiomar Cuesta. Comps. Bogotá: Ministerio de Cultura, 2008.276-285.

Geisdorfer Feal, Rosemary. "Feminism and Afro-Hispanism: The Double Bind". Afro-Hispanic Review 10. 1 (1991): 25-29.

Green Williams, Claudette Rose. "The Myth of Black Female Sexuality in Spanish Caribbean Poetry: A Deconstructive Critical View". Afro-Hispanic Review 12.1 (1993): 16-23.

Grueso Romero, Mary. "Negra soy". „ Negras somos! Antología de 21 poetas afrocolombianas de la región Pacífica. Alfredo Ocampo y Guiomar Cuesta. Comps. Bogotá: Ministerio de Cultura, 2008. 157-158.

— . "Orishas". ¡ Negras somos! Antología de 21 poetas afrocolombianas de la región Pacífica. Alfredo Ocampo y Guiomar Cuesta. Comps. Bogotá: Ministerio de Cultura, 2008. 167. 
- . "Zumbo Zurungo". ;Negras somos! Antología de 21 poetas afrocolombianas de la región Pacífica. Alfredo Ocampo y Guiomar Cuesta. Comps. Bogotá: Ministerio de Cultura, 2008. 161-162.

Johnson Reagan, Bernice. "African Diaspora Women: The Making of Cultural Workers". Women in Africa and the African Diaspora. Rosalyn Terborg-Penn et al. Eds. Howard University Press, 1989. 167-180.

Jones, Patrina. "African American Women's Nineteenth Century Public (Vocal) and Private (Text) Voice". Fournal of African Literature Association 6.2 (2011- 2012): 153-167.

Lavou Zoungbo, Victorien. Outsidering. Liminalité des noir-e-s Amériques-Caraïbes. Un hommage à Aimé Césaire. Persignan: Presses Universitaires de Persignan, 2007.

Lavrin, Asunción. "La mujer en la sociedad colonial latinoamericana". Historia de América latina. Ed. Leslie Bethell. Barcelona: Editorial Crítica, 1990.

Mina Diáz, Ana Teresa. "Cuerpo erótico”. ¡Negras somos! Antología de 21 poetas afrocolombianas de la región Pacífica. Alfredo Ocampo y Guiomar Cuesta. Comps. Bogotá: Ministerio de Cultura, 2008. 187. Montes del Valle, Agripina. "Proyectos de literatura". El Oasis 40 (1868): 314-316. —. "Contestación". El Oasis, Medellín, Serie II, Trimestre I, 1869, núm. 5: 37-38

Moreno Aguirre, Dionicia. "Recobrando el pasado". ¡Negras somos! Antología de 21 poetas afrocolombianas de la región Pacífica. Alfredo Ocampo y Guiomar Cuesta. Comps. Bogotá: Ministerio de Cultura, 2008. 359.

Muñoz, Willy. Polifonía de la marginalidad. La narrativa de escritoras latinoamericanas. Santiago de Chile: Editorial Cuarto Propio, 1999.

Navia Velasco, Carmina. "Historia de la literatura y estudios de género". Coords. Olga Vallejo Murcia y Alfredo Laverde Ospina. Visión histórica de la literatura colombiana. Elementos para la discusión. Medellín: La Carreta Editores, 2009. 159-172.

Panchano, Lucrecia. "Carimba". iNegras somos! Antología de 21 poetas afrocolombianas de la región Pacífica. Alfredo Ocampo y Guiomar Cuesta. Comps. Bogotá: Ministerio de Cultura, 2008. 103-108.

Posada de Pupo, Elisa. "Forma de vida”. ¡Negras somos! Antología de 21 poetas afrocolombianas de la región Pacífica. Alfredo Ocampo y Guiomar Cuesta. Comps. Bogotá: Ministerio de Cultura, 2008. 93

Prada Orepeza, Renato. El discurso testimonio y otros ensayos. México: Difusión Cultural UNAM, 2001.

Ramírez, María Teresa. "Dinga y Mandinga". ¡Negras somos! Antología de 21 poetas afrocolombianas de la región Pacífica. Alfredo Ocampo y Guiomar Cuesta. Comps. Bogotá: Ministerio de Cultura, 2008. 129-130. 
Randall, Margaret. "¿Qué es, y cómo se hace un testimonio?”. Revista de Crítica Literaria Latinoamericana 18.35 (1992): 21-45.

Rice Murray, Sandra. "Psychological Research Methods: Women in the African Diaspora". Women in Africa and the African Diaspora. Rosalyn Terborg-Penn et al. Eds. Howard University Press, 1989 - 79-95.

Rosada de Pupo, Elisa. "Posesión". iNegras somos! Antología de 21 poetas afrocolombianas de la región Pacífica. Alfredo Ocampo y Guiomar Cuesta. Comps. Bogotá: Ministerio de Cultura, 2008. 95

Said, Edward. "An Exchange on Deconstruction and History". Boundary 2-8.1 (1979): 65-74.

Terborg-Penn, Rosalyn et al. Women in Africa and the African Diaspora. Washington, DC, 1989.

Torres Herrera, Lorena. “Siempre presentes”. „Negras somos! Antología de 21 poetas afrocolombianas de la región Pacífica. Alfredo Ocampo y Guiomar Cuesta. Comps. Bogotá: Ministerio de Cultura, 2008. 427-429.

Vargas, Margarita. "Introduction". Women Writing Women. Anthology of Spanish-American Theater of 1980s. Eds. Teresa Cajiao Salas y Margarita Vargas. Albany: SUNY Press, 1997: 1-17.

Welles, Marcia L. "The Changing Face of Women in Latin American Fiction". Shame in Context. Susan Beth Miller, Ed. Hillsdale, NJ: Analytic Press, 1996. 280-288. 